\title{
Participación en la formulación de políticas culturales. El caso del Consejo Nacional de la Cultura y las Artes (CNCA) durante 2011-2016
}

\author{
Paola Moreno
}

Univesidad de Chile

\section{Resumen}

El objetivo de esta investigación es analizar la trayectoria de los instrumentos de participación en el proceso de formulación de políticas públicas culturales 2011- 2016, identificando si hay cambios en los mecanismos institucionales y normativos que aplica el Estado para implementar los principios orientadores de las políticas culturales en Chile, además de establecer sus actores y procedimientos. El estudio de caso es de tipo exploratorio descriptivo, con uso de metodología cualitativa, mediante el análisis de documentación secundaria institucional. Se concluye que el cambio de signo político del gobierno de turno impacta en la orientación de las políticas en cultura. Asimismo existe un desempeño limitado de la participación de la sociedad civil en el Directorio, pudiéndose avanzar tanto el fortalecimiento de la participación como en la generación de mecanismos que promuevan una mayor apertura de la Administración, a través de la incorporación del ciudadano no organizado, creando una red más abierta y plural, aumentando así el carácter democrático de la relación Estado-Sociedad Civil.

Palabras clave: Políticas culturales, gobernanza, formulación de políticas públicas.

Participation in the cultural policies formulation. The case of the National Council of Culture and the Arts (CNCA) during 2011-2016

\begin{abstract}
This research objective is to analyze the trajectory of participations instruments in the formulation of cultural public policies 2011- 2016, identifying changes in institutional and regulatory arrangements applied by the State to implement the guiding principles of cultural policies in Chile, also establishing its actors and procedures. The study case is descriptive exploratory, using qualitative methodology, analyzing institutional supporting documentation. It is concluded that the change of government impacts the orientation of policies on culture. There is also a limited participation of civil society in the directory, leaving space to improve both the strengthening of participation and the creation of mechanisms to promote more open government, through the incorporation of citizen unorganized, creating a more open and plural network, thus increasing the democratic nature of the state-civil society relationship.
\end{abstract}

Keywords: Cultural policies, governance, public policies formulation.

*Dirección de correspondencia [Correspondence

address]: Paola Moreno, Univesidad de Chile

E-mail: paolamorenoh@gmail.com 


\section{Introducción}

Durante las dos últimas décadas, nuestro país enfrentó cambios muy profundos, dando lugar a una sociedad más presente en los asuntos públicos, más informada y protagónica, lo que plantea un desafío para una nueva forma de gobernar, que integre a la sociedad civil, más demandante, en los asuntos públicos que antes eran concebidos solo para el Estado.

Una de las iniciativas que integra la participación en la formulación de las políticas públicas, recogiendo las necesidades de una ciudadanía más participativa, es la realizada con la creación del CNCA, en el marco del Gobierno del Presidente Lagos (20002006), dentro de su proyecto de reforma y modernización del Estado. Esta institución fue creada por la ley 19.891 de 2003, y destaca por ser una innovación institucional, ya que a diferencia de la gran mayoría de organismos públicos que tienen una estructura vertical en la elaboración de políticas públicas, el CNCA contempla la participación de la sociedad civil en su estructura orgánica. Dicha estructura está encabezada por un órgano colegiado de dirección superior denominado Directorio Nacional, integrado por cinco personalidades destacadas del ámbito de la cultura, dos académicos y un Premio Nacional. Los representantes estatales están conformados por el presidente quien tiene el rango legal de Ministro de Estado, el Ministro de Educación y El Ministro de Relaciones Exteriores.

La creación de esta institucionalidad se enmarca en las iniciativas que se suceden a partir del año 2000 , con la llegada de dicho gobierno y su objetivo de concretar una reforma que permita la participación ciudadana en la gestión pública. Así, en diciembre de 2000, el gobierno chileno inició un proyecto cuyo propósito era mejorar los mecanismos de participación ciudadana, tanto en el interior de la gestión pública como en la asociatividad con el sector civil, buscando en un principio, que las políticas y programas públicos incidieran en una mejor gobernabilidad y consolidación del sistema democrático, estimulando una sociedad proactiva que comparte un proyecto país con el Estado (Gentes, 2006). Esta línea se consolida con la promulgación de la ley 20.500 de 2011, que establece el derecho de las personas a participar en los distintos espacios de la gestión pública, acercando a los gobiernos locales y la administración central a la ciudadanía. La ley busca fortalecer la participación regulando las organizaciones y asociaciones para incentivar la participación en la gestión pública, especialmente en el ámbito local.

Esta visión inclusiva de la sociedad civil y su par- ticipación, específicamente en el caso del CNCA, es el tema central del presente estudio, el cual será analizado desde el concepto de gobernanza, que incorpora la perspectiva de la participación, como el trabajo en red, específicamente del gobierno multinivel. Esta opción analítica es pertinente pues estos preceptos se reflejan en el CNCA en términos de sus órganos participativos como en el Directorio, el cual es asesorado además por un Comité Consultivo Nacional; modelo que se replica a nivel regional, mediante los Consejos Regionales presididos por un Director/ra Regional, asesorado por un Comité Consultivo Regional.

El CNCA es uno de los servicios públicos que mejor representa la integración de la sociedad civil en el diseño y control de políticas públicas en sus ámbitos de competencia, aspirando a la gestión de políticas concebida desde una perspectiva más amplia, pasando de política de gobierno a política de Estado (Arntz y Soto, 2009).

En específico, la arquitectura de la política cultural es impulsada por la función primera que tiene el CNCA de "estudiar, adoptar, poner en ejecución, evaluar y renovar políticas culturales", la cuestión es dar cuenta de los actuales desafíos del Chile de hoy en el ámbito del resguardo y difusión del patrimonio, de la protección de las culturas originarias, del apoyo a la creación artística, y del desarrollo de las audiencias, los mercados y las industrias culturales (Consejo Nacional de la Cultura y las Artes, 2005). La política cultural se trata de una política de Estado que, como tal, concierne tanto a los agentes públicos como privados que operan en el ámbito cultural.

A partir de esta mirada, el CNCA pretende desde su primer año de creación, implementar un esquema de acción participativa e inclusiva que permita sumar e integrar los aportes de los distintos actores del ámbito cultural. Se elaboran así las definiciones de política cultural 2005-2010, mediante el documento "Chile quiere más cultura". Esta "carta de navegación", resultó de un trabajo coordinado por el Directorio del CNCA y contó, gracias a la Convención Cultural del 2004, con la participación de los representantes del mundo cultural (Muñoz del Campo, 2011).

Por otra parte, los compromisos asumidos en el documento "Chile quiere más cultura", demandarán acciones no sólo del Consejo Nacional de la Cultura y las Artes, sino también de otros servicios, organismos públicos y ministerios, lo cual hará necesaria una debida y permanente coordinación entre todos ellos. El documento constituye también un referente importante para las políticas y acciones de los agentes y organismos privados que trabajan en 
la cultura.

Considerando lo anterior, resulta relevante analizar el proceso de formulación de políticas públicas culturales 2011-2016, identificando qué mecanismos institucionales y normativos provee y aplica el Estado para implementar los principios orientadores de las políticas culturales en Chile y definir los mecanismos de participación. En torno a ello es que se definen las preguntas directrices de la investigación en torno a ¿el proceso de elaboración de políticas culturales 2011-2016 asume y promueve efectivamente instancias para el diálogo y la deliberación del contenido de las mismas? ¿La administración se muestra dispuesta a escuchar y a responder al resto de los actores?

De esta forma, el presente trabajo de investigación busca analizar los mecanismos de participación en el proceso de formulación de políticas públicas culturales que se dan en el Consejo Nacional de la Cultura y las Artes (CNCA) entre 2011 y 2016, identificando si hay cambios respecto de los mecanismos institucionales y normativos que aplica el Estado para implementar los principios orientadores de las Políticas Culturales en Chile, además de establecer los mecanismos de participación, sus actores y procedimientos.

\section{Gobernanza y participación en las políticas públicas}

La gobernanza es un modelo de gobierno en el que a diferencia del modelo jerárquico, en donde las autoridades estatales ejercen un poder soberano sobre la sociedad civil (Mayntz, 2001), existe una práctica cooperativa entre Estado y sociedad civil, a través intercambio, coordinación, control y adopción de decisiones conjuntas (Kooiman, 1993). Se puede definir, por lo tanto, como las relaciones entre el Estado y la sociedad civil con el fin de resolver problemas colectivos y lograr el bienestar público a través de la formulación de políticas públicas que incluyan de forma efectiva la participación ciudadana.

El fortalecimiento de la representatividad democrática pasa necesariamente por considerar la existencia de distintos actores, sean estos del ámbito social, político, gubernamental y económico, de tal manera de construir paulatinamente más y mejores espacios de coordinación y comunicación. Particularmente, la participación es importante en coyunturas en las cuales los mecanismos formales (elecciones, partidos políticos, militancia) se encuentran cuestionados (Jouve, 2004). Este escenario supone el reconocimiento de múltiples actores y niveles con intereses y recursos a aportar en las políticas públicas (Blanco y Gomá, 2006).

El estudio de la gobernanza es multidireccional y se ha asociado a diferentes ámbitos, modalidades de actores y niveles de gobierno (local, subnacional, nacional y supra nacional) con el fin de estudiar patrones, estructuras y reglas del juego que facilitan o limitan la articulación e interacción socio política (Natera, 2004).

La gobernanza resalta el carácter confuso de la separación entre el ámbito privado y público y resitúa las responsabilidades colectivas en un espacio compartido entre ambas. En este sentido, se hace referencia a como mínimo dos procesos simultáneos, no siempre fáciles de conciliar: por una parte la incorporación de organizaciones lucrativas, a través de su participación en foros públicos de deliberación y/o del establecimiento de relaciones de agencia para la resolución de problemas públicos; y por otra parte, la apertura de nuevos espacios de participación ciudadana. De esta manera, la gobernanza se encuentra ligada a la capacidad de influencia y liderazgo en el conjunto de una red que incorpora actores públicos y privados, mediante el incentivo al intercambio y a la cooperación entre diversos actores.

La gestión de redes se trabaja en dos niveles diferenciados:

- La gestión de las interacciones internas, a) activar la red; b) Fortalecer la densidad relacional, c) Fortalecer la intermediación.

- La gestión de la estructura de la red, a) Incidir sobre la configuración básica de la red; b) Incidir sobre las distribuciones de poder, c) Incidir sobre los valores y las percepciones.

Desde esta perspectiva, se puede concebir la política pública como un micro escenario diferenciado, poblado de estructuras político-administrativas, valores y actores específicos, públicos y privados, que se corresponde con el respectivo ámbito de problemas, necesidades y/o oportunidades; reconstruido por las características y el estilo de esa política pública (Vanaclocha, 2001).

\section{Modelos participativos de gestión e implementación de política pública}

Peters (1995) distingue entre dos modelos alternativos en el proceso de formulación de las políticas públicas: los modelos bottom up o ?de abajo hacia arriba? y top down o ?de arriba hacia abajo?. En Chile este proceso por lo general responde a una estructura top down o ?de arriba hacia 
abajo?, propia de los modelos tradicionales, donde los gobiernos actúan como únicos sujetos de acción pública, descansando su legitimidad en el imperio de la ley, estableciendo como responsable del proceso de elaboración, implementación y control de políticas públicas de carácter nacional a los Ministerios. Esto se debe a que el proyecto político de la transición contempló solamente la democratización política a nivel de los municipios y no un cambio en la estructura de la arquitectura institucional y administrativa del país (De la Maza, 2010).

Hablar de la participación de la sociedad en la construcción de políticas públicas es hablar de gobernanza, de responsabilidad social, de nuevas maneras de relacionar la administración con la ciudadanía. De esta forma, para las empresas, las organizaciones sociales y la ciudadanía, la participación en los procesos promovidos por las administraciones constituye igualmente una oportunidad de tener información, de contrastarla con la administración y con otros agentes económicos y sociales, de debatir los diferentes puntos de vista sobre los temas más problemáticos y de elevar propuestas para su consideración posterior. La participación pública sirve también para generar una nueva cultura favorable al diálogo, la colaboración y el compromiso activo de las administraciones, de la ciudadanía y sus organizaciones en la solución de los problemas colectivos.

Democratizar la administración mejora la eficiencia y la eficacia, ya que utilizar solo la capacidad técnica que aportan los profesionales del sector público supone exclusión. Canalizar la participación de los interesados no es simplemente una voluntad más o menos altruista, sino una necesidad funcional de las grandes organizaciones actuales. La legitimidad del proceso decisional es crucial en la medida que genera colaboración externa e interna. La democratización de la administración mejora la eficiencia porque los out a alcanzar son consensuados con los actores afectados y/o interesados en ellos. Asimismo facilitar el dialogo horizontal en el interior de la propia organización mejora los rendimientos administrativos (Brugué y Gallego, 2001).

Por último, democratizar la administración mejora el rendimiento institucional, que se ha definido como la capacidad de las instituciones públicas de dar respuestas a las necesidades sociales y de ser efectiva en sus actuaciones, esto es, en sus interacciones con la sociedad.

\section{La política nacional de cultura $2005-$ 2010}

El proceso de formulación de las políticas culturales 2005-2010 es el fruto de un esfuerzo colectivo encabezado por el Directorio Nacional del Consejo Nacional de la Cultura y las Artes (CNCA), que comprometió la participación de los trece Consejos Regionales y de los Comités Consultivos.

En el caso del CNCA, podemos apreciar una innovación en la forma de hacer gobierno, ya que el Mandato Presidencial descansa en una figura pluripersonal, la Dirección Superior del Consejo, el Directorio. Además se contempla el Comité Consultivo Nacional, órgano colegiado, encargado de asesorar al directorio en lo relativo a políticas culturales, plan anual de trabajo, y preparación de proyectos de ley y actos administrativos concernientes a cultura (art.12º de la ley 19.891), y que estará integrado por personas de reconocida trayectoria y experiencia en las distintas áreas de la creación artística, el patrimonio cultural, la actividad académica y la gestión cultural. Esta estructura se replica a nivel regional, ámbito en el cual se encuentran los Consejos Regionales y los Comités Consultivos, que consideran también la participación de la sociedad civil en la definición de las políticas regionales. Por último, encontramos los Consejos Sectoriales, que también contemplan representantes de la sociedad civil y están a cargo de definir las políticas pertinentes a cada ámbito de fomento cultural. En estos órganos principalmente está reflejada la participación en el proceso de formulación de políticas públicas en cultura, siendo por cierto el Directorio el único que tiene un carácter resolutivo.

Ahora bien en la línea de la implementación de políticas públicas, encontramos que a partir del Gobierno de Bachelet, se anunció públicamente la Agenda Pro Participación Ciudadana y posteriormente el Instructivo Presidencial para la participación ciudadana, que establecen el deber que tiene la Administración Pública de crear los mecanismos necesarios para que las personas incidan en el desarrollo de las políticas públicas que son de su competencia. En este contexto, el CNCA el año 2009, aprueba la norma general de participación ciudadana del Consejo Nacional de la Cultura y las Artes. Dicha Norma, establece 8 instancias de participación:

- Los Órganos Colegiados del Consejo.

- Los Consejos Sectoriales.

- Las Mesas Artísticas Regionales.

- Los Fondos de Cultura administrados por el CNCA. 
- Diagnósticos participativos:

- Comités Culturales Barriales del Programa Creando Chile en mi Barrio.

- Programa Chile quiere Más Cultura.

- Consulta Ciudadana:

- Consejos Escolares del Programa Okupa tiempo y espacio creativo en tu liceo.

- Jornadas de evaluación de las Fiestas Ciudadanas del Programa Chile quiere Más Cultura.

- La Cuenta Pública Anual.

- Los Sistemas de Información Ciudadana.

Estos mecanismos de participación refuerzan un modelo innovador de gobernar en el que se incorpora la sociedad civil en la definición e implementación de políticas, los distintos actores se reconocen como sujetos activos en el proceso político y de gestión y las políticas culturales tienden a desarrollarse en un enfoque de Gobernanza. Así también, la implementación de políticas responde más bien a un modelo bottom-up, mediante la creación de los Programas Creando Chile en mi Barrio y Chile quiere Más Cultura, que buscan recoger las demandas y necesidades de la sociedad desde las bases, incorporando sectores populares.

\section{Metodología}

Esta es una investigación descriptiva y exploratoria, estructurada a partir de un modelo de análisis de fuentes secundarias de tipo cualitativo. La primera aproximación se realiza a través de la consulta de los distintos documentos de acceso público, Balance de Gestión integral, sitio web del CNCA, la Dipres, las Memorias 2004-2010 de la Institución, las Convenciones Nacional de Culturas, las actas del CNCA (último semestre de 2011), las Políticas Culturales 2005-2010 y 2011-2016, el proceso de elaboración y su metodología, entre otros.

Se definieron cinco instancias de análisis a partir de esta recopilación:

- La normativa que regula el CNCA (Estructura, Órganos, Fondos)

- La normativa que regula el proceso de formulación de políticas culturales.

- Institucionalidad participativa.
- Instancias de participación en el proceso de formulación de políticas culturales 2011-2016.

- El posicionamiento e intereses de los actores participes del proceso.

Para la identificación de los actores relevantes dentro del proceso, se confeccionó un mapa de actores sociales, instrumento analítico que permitió identificar a todas las personas y organizaciones que pueden ser relevantes en el proceso de diseño e implementación de las políticas culturales chilenas durante el período 2011-2016. Se establecieron los actores individuales, grupales y organizaciones y las características relevantes de los mecanismos, herramientas e incidencia en el proceso de toma decisiones, su interés en la problemática, y la posición que podrían adoptar frente al tema en cuestión, para lo cual se utilizó el modelo de "Participation and Social Assessment: Tools and Techniques" (Rietbergen-McCracken y Narayan-Parker, 1998).

Por último, el análisis de la información obtenida en fuentes secundarias se realizó mediante un análisis de contenido, que pretende conducir a una descripción e interpretación fundada del contenido manifiesto (Krippendorff y Wolfson, 1990).

\section{Análisis del proceso de formula- ción de políticas culturales 2011 $-2016$}

Al revisar las políticas culturales 2011-2016, se puede desprender que efectivamente ha habido un ejercicio de participación importante. Según CruzCoke, Ministro Presidente del CNCA, el documento "Política Cultural 2011-2016", plasma el resultado de un proceso participativo, reflexivo y eminentemente inclusivo, inspirado en las dinámicas transversales, representativas, descentralizadas y pluralistas que representan el espíritu del Consejo Nacional de la Cultura y las Artes. Según el Directorio, este documento es el resultado de un esfuerzo amplio, democrático, altamente participativo, comprometido y riguroso, que apunta a plasmar en directrices y líneas de acción concretas los anhelos de la mayoría de los chilenos abocados al perfeccionamiento del desarrollo de la cultura y las artes.

A partir del 2003 se institucionalizó la Cultura y las Artes mediante la creación del CNCA, distinguiéndose dos instancias de formulación o elaboración de políticas públicas culturales, siendo la primera el año 2005-2010 y la segunda 2011-2016. Si bien ambos procesos de política pública están marcados por la participación ciudadana, en el segundo 
período se modificaron las instancias participativas existentes, en términos de eliminar algunas, incorporar nuevas y mantener otras, siendo los principales cambios los siguientes:

Se eliminan:

- Diagnósticos participativos:

- Comités Culturales Barriales del Programa Creando Chile en mi Barrio.

- Programa Chile quiere Más Cultura.

- Consulta Ciudadana:

- Consejos Escolares del Programa Okupa tiempo y espacio creativo en tu liceo.

- Jornadas de evaluación de las Fiestas Ciudadanas del Programa Chile quiere Más Cultura.

- Las Mesas Artísticas Regionales.

Se incorporan:

- Los Grupos Focales

- La Consulta Ciudadana vía Web.

- La propuesta cultural de la Unión Nacional de Artistas

Se mantienen:

- Los Órganos Colegiados del Consejo.

- Los Consejos Sectoriales.

- Los Fondos de Cultura administrados por el CNCA.

- La Cuenta Pública Anual.

- Los Sistemas de Información Ciudadana.

En lo relativo a los mecanismos de participación, en el segundo período se eliminaron los mecanismos que fomentaban un diálogo directo con los actores del ámbito local, ya que los diálogos participativos se dan entre los expertos, los creadores, artistas, los organismos propios de la institucionalidad, los grupos organizados y los grupos de influencia.

En cuanto a los otros elementos característicos de este período, se puede apreciar que el énfasis esta puesto en la Modernización Institucional, Mejoramiento en la gestión, en la Operacionalización de las políticas Culturales y en la Conformación de redes y alianzas entre el sector privado y público, fomentando el financiamiento conjunto.

\section{Normativa que regula el proceso}

Según lo establecido en la Norma de aplicación general sobre participación ciudadana en el CNCA (Resolución Exenta № 3695 , del Consejo Nacional de la Cultura y las Artes, de 2011), los mecanismos de participación ciudadana en el CNCA constituyen instancias de diálogo constante y fluido entre este organismo y los miembros activos de la ciudadanía, actúen agrupados o individualmente. En concordancia con lo anterior, se constituyen las siguientes modalidades y formas específicas de participación ciudadana en el Consejo:

- Cuenta Pública Participativa: A. El Ministro Presidente del Consejo realizará una Cuenta Pública Anual dentro del segundo semestre de cada año. El CNCA convocará a distintos representantes de la sociedad civil a participar en esta instancia, en la que se expondrá de manera clara el contenido de la Cuenta Pública. En la oportunidad se tomará acta de las consultas, comentarios y opiniones generados en esta instancia. B. Los Consejos Regionales de la Cultura y las Artes, anualmente darán cuenta pública participativa a la ciudadanía de la gestión de sus políticas, planes, programas, acciones y de su ejecución presupuestaria, con la finalidad de informar a la ciudadanía sobre la gestión realizada, recoger preguntas y planteamientos de la ciudadanía y dar respuestas a estas.

- Consejos de la Sociedad Civil: El CNCA cuenta con Consejos de la Sociedad Civil de carácter consultivo y autónomo, a excepción del Directorio Nacional que tiene carácter resolutivo, que tienen como objetivo acompañar los procesos de toma de decisiones y seguimiento de las políticas públicas impulsadas por el Servicio. Los órganos colegiados son:

- Directorio Nacional

- Comité Consultivo Nacional

- Consejos Regionales de la Cultura y las Artes

- Comité Consultivo Regionales

- Consejeros Sectoriales: Consejo del Arte y la Industria Audiovisual, Consejo de Fomento de la Música Nacional y Consejo Nacional del Libro y la Lectura.

- Consulta Ciudadana: El CNCA, dentro de su competencia, de oficio o a petición de parte, deberá señalar aquellas materias en que se requiera conocer la opinión de las personas, implementando para dichos efectos la autoridad 
los espacios y canales de consulta para invitar a la ciudadanía a participar de manera informada, pluralista y representativa, entregando una respuesta oportuna y de calidad en las áreas de su competencia. La consulta a la ciudadanía, podrá ser realizada utilizando cualquiera de las Modalidades que a continuación se individualizan, u otros que para caso se determine:

- Ventanillas Virtuales de Opinión

- Diálogos Participativos

- Convención Nacional de Cultura

- Convenciones Zonales de Cultura

- Acceso a la Información Relevante: El Consejo pondrá en conocimiento público información relevante acerca de sus políticas, planes, programas, acciones y presupuestos asegurando que esta sea oportuna, completa y ampliamente accesible. Toda información que el Consejo considere relevante será publicada en el sitio electrónico del Consejo, de manera que pueda ser conocida por la ciudadanía, la cual podrá efectuar consultas y sugerencias a través de los canales de atención de público coordinados a través del Sistema Integral de Información Ciudadana (SIAC).

El CNCA mediante la Resolución Exenta № 3582, de 2011, modifica la estructura interna del servicio ampliando las funciones de la Sección de Estudios e Investigación Cultural, entregándole competencias propias, específicas y niveles dependientes que las desarrollarán, además de dejar de ser una Sección del Departamento de Planificación y Presupuesto, pasando a ser un Departamento especialmente creado para analizar el proceso de las Políticas públicas culturales.

\section{Institucionalidad de participación del CNCA}

Según, Martínez Villarroel (2004), los consejos ciudadanos constituyen uno de los instrumentos más utilizados para formalizar la intervención de actores no gubernamentales en distintas fases del proceso de políticas, ya sea en gobiernos nacionales, estatales o municipales. Hay diferentes opciones de diseño institucional de los consejos ciudadanos, que dependen de distintas dimensiones: integración, representación de consejeros ciudadanos, mecanismos de selección de consejeros ciudadanos, permanencia en el cargo, presidencia, atribuciones de consejeros ciudadanos y funcionamiento. Las dimensiones son caracterizadas mediante ciertos atributos que permiten concluir una mayor democratización de los consejos versus una mayor centralización de estos en razón de la configuración que adoptan.

Para el CNCA, su configuración es analizada en base a su integración, representación, mecanismos de selección de sus miembros, permanencia en los cargos, su presidencia y atribuciones.

- Integración, con respecto a este elemento se pudo observar que la composición de la Dirección Superior del CNCA, está integrada por 11 miembros, tres por derecho propio y ocho designados. El Jefe del Servicio y dos Ministros de Estado, son funcionarios y 8 representantes de la Sociedad Civil, por lo tanto la mayoría correspondería a ciudadanos.

- Representación de consejeros ciudadanos, al analizar este elemento, podemos observar que no está incorporada la participación individual ni tampoco la participación social, por lo tanto podríamos concluir que la representación se encuentra limitada a los tecnócratas, los expertos y los grupos de influencia.

- Mecanismos de selección de consejeros ciudadanos, al observar este atributo, podemos apreciar que los miembros del CNCA son designados, cinco de ellos por el Presidente de la República, de los cuales tres a propuesta de las organizaciones culturales del país que posean personalidad jurídica vigente y estén inscritas en el Registro Nacional que llevará el Consejo. Por lo que la participación de los ciudadanos se manifiesta de manera colectiva, pero restringida a la participación de las organizaciones creadas por iniciativa estatal. Las otras dos personalidades, serán designadas con acuerdo del Senado. Los dos académicos, designados uno por el Consejo de rectores de las Universidades Chilenas y otro por los Rectores de las Universidades Privadas autónomas. Un galardonado por el premio nacional, elegido por quienes hayan recibido tal distinción. En este sentido podríamos concluir que el mecanismo de selección podría ser bastante más participativo, ya que los miembros son designados por autoridades gubernamentales o bien por grupos de interés no siendo elegidos por la ciudadanía, lo que restringe el nivel de representatividad o participación de este Órgano Colegiado. Así también al ser la mayoría de los miembros de la Sociedad Civil designados por el Presidente de la República, difícilmente este designará miembros contrarios a sus ideas país, independiente que no coincida el período del gobierno de turno, con el ejercicio del cargo de los Consejeros, esta situación se agudiza con el hecho de que el Jefe de Servicio es eminen- 
temente un cargo político que obedece instrucciones y debe ejecutar el mandato presidencial. En cuanto a los nombramientos de los demás Órganos colegiados y Consejos Sectoriales de la sociedad Civil, el Directorio observa la forma en que se designan reclamando una mayor injerencia en la propuesta de personas para la designación del Presidente.

- Permanencia en el Cargo, en este caso está dado por un criterio de cuatro años, no incorporando criterios de desempeño en su mantención o desvinculación.

- Presidencia el Ministro Presidente, cumple las funciones de Jefe de Servicio y Presidente del Consejo. Esta condición hace que efectivamente sea difícil delimitar el cruce de competencias entre sus atribuciones y las de este Directorio, como lo señala el Ministro de Cultura Cruz Coke, durante 2010-2013, quien expresa que para un buen funcionamiento, el Directorio debe ser decisorio, sólo respecto de políticas públicas, sin intervenir en otras materias más específicas. Esta afirmación se da como una crítica ya que la ley contempla bastante más atribuciones propias del Directorio. Más aún, este órgano contempla que las decisiones deben ser adoptadas de forma informada y consultada al organismo colegiado, lo que limita las atribuciones del Ministro y del Directorio, impulsando la participación en las decisiones

- Atribuciones de consejeros ciudadanos, por normativa son amplias, la única que no se contemplaría es la de aplicación de sanciones; no obstante, en la práctica las actas del Directorio revelan que no existe consenso entre sus miembros con respecto a las facultades y atribuciones.

Al observar las distintas intervenciones de los miembros del Directorio versus los funcionarios del CNCA, se evidencian distintas tensiones en la línea de:

- Las Facultades y atribuciones del Directorio V/S las facultades del Ministro Presidente.

- En las Comunicaciones, internas y externas.

- En el Manejo de la información

- En la confianza entre sus miembros

- En el liderazgo del Ministro presidente V/S el Directorio.

Podemos apreciar entonces, el gran poder e influencia que tiene el Ministro Presidente dentro del
CNCA, que responde más bien a comportamientos propios de la burocracia tradicional, versus el resto de los actores involucrados, que están permanentemente abogando por sus facultades y atribuciones, en las distintas materias, que por ley les corresponde abordar.

\section{Resultados}

A partir del análisis de las actas de las sesiones del Directorio Nacional, se puede evidenciar dos visiones del proceso de formulación de política pública, una top-down y otra bottom-up.

\section{Visión top-down}

Para efectos de esta investigación se visualizan ciertos hitos que evidencian esta postura: en abril y mayo de 2010, el Ministro Presidente da a conocer al "Directorio Nacional" los lineamientos estratégicos 2010-2014, que se presentarían en el discurso del 21 de mayo, y expone varias de las medidas y políticas culturales del Programa de Gobierno. Esta situación refleja la naturaleza más lineal y autoritaria que guiarán su administración, además de una mirada centralista con respecto al tema del Patrimonio y la reconstrucción. En este contexto, se produjo una discusión acerca de la institucionalidad cultural que debiese existir en Cultura, en cuanto a ser Ministerio o a mantenerse como Consejo. Frente a este planteamiento se evidencian dos posiciones: una representada por el Ministro, quien preside el CNCA, y dos de sus Directores, quienes plantean la necesidad de que el Consejo pase a ser un Ministerio, apelando a su autonomía y eficiencia. Por otra parte, la otra posición del Directorio Nacional, integrada por 5 representantes de la sociedad civil, la academia y los premios nacionales, propone que el Consejo debe mantener su calidad institucional, ya que de transformarse en Ministerio perdería capacidad para convocar participación de la sociedad civil, lo cual es un elemento básico y fundamental en el funcionamiento del Consejo.

En noviembre de 2010, el Ministro Presidente expone los cinco ejes del plan de gobierno y dos ejes transversales: a) Modernización; b) Fomento de las Audiencias; c) Fortalecimiento de Industrias; d) Educación y Cultura; y e) Infraestructura y Gestión Cultural.

En diciembre de 2010, el jefe del Departamento de planificación y presupuesto, presenta la Propuesta de plan de trabajo 2011. Para esta propuesta se usaron como insumos: Los insumos que han utiliza- 
do para su elaboración: cuenta pública, prioridades de departamentos y directores regionales y programa de Gobierno. Los directores comentan que los insumos utilizados tienen solo una fuente, esto es la Nueva administración representada por el Ministro Presidente. Por otro lado, los directores indican que si bien no es posible utilizar las políticas como insumo por no estar aprobadas, sí deben utilizar el material que se ha ocupado en su elaboración, esto es, los resultados de las convenciones zonales, la convención Nacional y de los focus groups.

A partir de esta intervención se revela una tensión entre la mayor disposición del Directorio a participar y una lógica más centralizada top-down del Ministro. Observamos que el Directorio aboga porque el Plan de trabajo año 2011 esté constituido en base a un enfoque más bottom-up, contemplando las necesidades emanadas de las distintas instancias de participación desarrolladas.

En mayo de 2011, durante el ejercicio de la Cuenta Pública del 21 de mayo, el Presidente anuncia un cambio en la institucionalidad de la cultura. Antes de este anuncio la mayoría de los miembros del Directorio CNCA rechazaban la iniciativa y el no haber sido participe de esta. Sin embargo, transcurrido un año desde la primera intervención del Ministro Presidente en la cual da a conocer al "Directorio Nacional" los lineamientos estratégicos 20102014, se observa que las posiciones originales de los miembros del Directorio ante una Nueva institucionalidad Cultural cambiaron, arguyendo que existen argumentos sólidos para la creación del Ministerio y destacando la importancia del rol de la sociedad civil en este. En general se puede concluir que los miembros del Directorio comparten la posición del Ministro Presidente, no obstante enfatizan en la necesidad de mantener la representación de la sociedad civil en la nueva Institucionalidad y que esta incida de manera vinculante en la definición de las políticas culturales.

\section{Visión de bottom-up}

Esta visión se construye a partir de la revisión de los distintos documentos resultantes del proceso de levantamiento de información de las instancias de participación para la construcción de las políticas culturales 2011-2016 ${ }^{1}$.

\footnotetext{
${ }^{1}$ Documentos proporcionados por la Jefa de estudios del CNCA: Conclusiones Levantamiento Modalidad de Trabajo "Mesas" VII Convención Nacional "sigamos Construyendo Futuro", agosto 2010; Informe Ejecutivo Final de la generación de la Política Pública 2011-2016 de la Cultura y las Artes del Gobierno de Chile, Algunos Alcances para la formulación de la nueva política pública cultural, Ideas a partir de los Grupos Focales con Agentes socio culturales, enero de 2011; Informe de Resultados Consulta Nacional de Políticas
}

Las distintas instancias de participación organizadas son instancias de participación de base, desde los afectados de las políticas, se desarrollan a nivel nacional, y constituyen instancias representativas de los distintos sectores que componen el ámbito cultura, sean estos del ámbito público, privado o de la sociedad civil.

Para el análisis de los contenidos de las instancias de participación están se clasifican en cuatro ámbitos:

\section{- Rol del Estado}

- Incidencia de la participación en las políticas de cultura

- Transparencia, información y acceso (oportuna, completa y ampliamente accesible).

- Financiamiento

\section{Rol del Estado}

Desde el Ministerio de Cultura, el rol del Estado en cultura se valora en términos de su aporte al desarrollo social y en la transformación de zonas con alta vulnerabilidad. De este modo, el Estado asume un rol en cultura que es una herramienta coadyudante al rol de otros Ministerios y servicios. Hay una preocupación por el trabajo en red y las competencias del propio Ministerio que deben fortalecerse en términos de financiamiento y subvenciones para el desarrollo de infraestructura cultural, así como la concentración de acciones en el ámbito de cultura en el Ministerio, potenciando su acción.

Por otra parte, la visión del rol del Estado extraída desde los documentos de la Convención Nacional, permiten establecer que el énfasis es diferente al del Ministerio, pues la preocupación central es el soporte a la gestión y potenciación del artista. Desde las mesas de trabajo se desprende el reclamo por una mayor presencia territorial del Ministerio y de políticas que resguarden la cultura territorial, potenciando la vinculación entre turismo, cultura y educación. Hay también una demanda de mayor participación en la CNCA, a través de mesas de participación. Sin embargo, la apuesta es el fortalecimiento del CNCA, que debe ser fortalecido con mayores recursos y atribuciones, siendo más beneficioso que la creación de un Ministerio de Cultura.

De la misma manera a partir de los resultados de la Convención Nacional se desprende que el prin-

Culturales Opino por Cultura, marzo 2011; Política cultural 2011-2016: La voz de los artistas, diciembre 2010; Cuenta Pública, Ministro Presidente, agosto 2010; Problemas detectados en la convenciones Zonales, julio-agosto 2010. 
cipal problema del CNCA es su centralización, por lo que muchas políticas solo se aplican en las capitales, y las provincias quedan rezagadas. El CNCA genera las políticas a partir de los consejeros y no de la comunidad propiamente tal (Mesas eje participación ciudadana). Se reconoce que la formación del CNCA ha permitido mayor autonomía respecto de las decisiones, disponer de mayores recursos y crear más espacios dedicados al desarrollo cultural, pero esto es insuficiente. Se propone fortalecer el CNCA, e incluso sería más beneficiosa la creación de un Ministerio de cultura, que permita cubrir toda la diversidad de nuestro país a través de los seremis.

Por su parte, desde la Unión de artistas, extraída a partir del documento "Política cultural 2011-2016: La voz de los artistas" (Consejo Nacional de la Cultura y las Artes, 2010), se desprende una interpelación al Estado para que resguarde y potencie a los artistas en dos grandes áreas: 1) el financiamiento de iniciativas artísticas a través de mayores recursos y fondos concursables (con líneas claramente diferenciadas para los artistas consagrados y para los emergentes); y 2) la protección a los trabajadores del arte, promoviendo una modificación a la ley de trabajadores de las artes y el espectáculo (Ley № 19.889 ).

A su vez, en los grupos focales se aprecia una posición que valora fuertemente el rol del Estado y exige una mayor participación de este en la actividad cultural. Se busca reducir la dispersión de organismos públicos en cultura (especialmente en los temas de patrimonio y propiedad intelectual) y una mayor descentralización. Se muestran críticas a la relación cultura-privados-economía-mercado, y se enfatiza que es necesario que el Estado asuma un rol de resguardo de la cultura, planteando como alternativa el financiamiento conjunto. Con respecto a la institucionalidad cultural Ministerio versus Consejo, no se observa consenso. Unos abogan a la creación de un Ministerio de la Cultura, atribuyendo que esto no se ha realizado debido a falta de voluntad de los consejeros. La otra tendencia es de mantener el consejo de la cultura, ya que se rescata y valora el que se constituya como un cuerpo colegiado. Asimismo, surge una posición intermedia donde se propone la creación de un ministerio que no pierda la lógica participativa y pluralista del consejo. Una cuarta visión en relación a este tema es que no interesa si es consejo o ministerio, sino que lo importante es el aseguramiento de los principios fundamentales como la participación. Se enfatiza en que las dimensiones culturales se deben trabajar en red, generando mecanismos de integración y fortaleciendo la credibilidad y confianza del CNCA. Se le atribuye al Estado el rol de la creación de una red de alianzas. En este sentido, el CNCA debe fomentar la inversión de privados nacionales y extranjeros, entre otras cosas porque la cultura es una actividad económica en crecimiento que puede aportar al PIB del país.

Por último, las convenciones Zonales enfatizan lo débil de la política de fomento a las industrias creativas a nivel nacional y regional. Destaca la necesidad de dar mayor seguimiento, evaluación y control a las políticas referidas a economía y cultura. Así también, en estas convenciones se reconoce mayoritariamente que las experiencias regionales son particulares y diversas las que han avanzado en la medida de sus posibilidades solicitando la colaboración de la Dirección Nacional, de las universidades locales o encargando estudios propios.

\section{Incidencia de la participación en las políticas de cultura}

Desde el Gobierno se enfatiza una mayor participación de regiones, mediante la creación de polos de desarrollo cultural regional, así como impulsar el consumo cultural constante en los sectores más vulnerables de nuestra sociedad.

Por otra parte, la visión de la incidencia de la participación extraída desde los documentos de la Convención Nacional, permiten establecer que el énfasis es diferente al del Gobierno. Este está puesto en generar políticas pertinentes a cada región, propiciando el intercambio cultural entre estas, así como el fortalecimiento y difusión de sus expresiones culturales aumentando así el gasto público en cultura, dado que se requiere robustecer los medios locales de comunicación y difusión, así como los medios nacionales que difundan la cultura local, generando audiencias y mayor interés comunitario incorporando a la comunidad en las decisiones que se toman a nivel de Directorio. Reformulando así la manera en que se generan las políticas y programas culturales, partiendo desde la comunidad y sus intereses y no hacia ellos. Generando espacios públicos regionales para la masificación y difusión de la cultura, arte y el patrimonio. Así también se aboga por el fortalecimiento de la institucionalidad regional en sus dimensiones administrativas y de gestión. La necesidad de mayor espacio para la cultura en la televisión abierta, donde se muestre el quehacer de cada una de las regiones. Dicho espacio debiese estar administrado por el CNCA.

Por su parte, los Grupos Focales distinguen tres principios fundamentales, en lo relativo a la incidencia en la participación: la democracia, la participación y la inclusión. Se enfatiza la creación de espacios participativos.

Desde la Unión de Artistas abogan por propiciar 
la real participación de las asociaciones artísticas y culturales nacionales en la formulación de las políticas culturales públicas.

\section{Transparencia, información y acceso (opor-} tuna, completa y ampliamente accesible)

Desde el Jefe de Servicio se busca ampliar la cobertura en cultura y revertir la brecha de desigual acceso cultural, se plantea otorgar un subsidio a la demanda cultural de grandes alcances, focalizándose en el público con menos posibilidades de acceso a los bienes culturales.

En los grupos focales, a diferencia del Ministro Presidente, el énfasis esta puesto en una política transparente en cultura, que promueva la igualdad de oportunidades. Asimismo, objetan la concursabilidad como el único mecanismo válido para la selección y adjudicación de fondos. También se critica la poca transparencia en asignación del aporte de la presidencia a través de los gastos reservados, ya que cuadruplican, los recursos de los fondos concursables.

Así también los grupos focales critican que los medios de comunicación, utilizados para informar y educar masivamente no ofrecen una oferta diversificada, tampoco son espacios que en los cuales se promueve la cultura, siendo estos una oportunidad para propiciar procesos reflexivos. También se aboga por una mayor regulación de las autoridades culturales acerca de los contenidos que surgen en estos medios y cómo estos además son herramientas del consumo.

\section{Financiamiento}

En materia de financiamiento, el Gobierno enfatiza las alianzas con los privados y las autoridades regionales locales, abogan por una corresponsabilidad en el desarrollo social, siendo el eje principal mejorar la Ley de Donaciones Culturales.

Desde la Convención Nacional se aboga por universalizar el acceso a la cultura, incorporando mayores recursos especialmente para regiones y la gratuidad para los sectores más pobres.

Desde los grupos focales se enfatiza que el presupuesto destinado a cultura es escaso sobre todo en comparación a las otras reparticiones públicas. También existe un malestar de los participantes en cuanto a la poca voluntad política que ha existido para realizar modificaciones legales y tributarias en el ámbito cultural señalando que se debe cambiar la Ley de Donaciones.
Así también en los grupos focales la discusión en torno al ámbito del financiamiento gira en torno a cuatro ejes fundamentales:

- La gestión cultural. Que consiste en mejorar la capacidad de articular redes para canalizar recursos económicos, tanto del sector privado como del Estado, con el fin de invertir en cultura en un amplio sentido.

- Canalización de Recursos para invertir en cultura. Se considera controversial la asignación de presupuestos que tiene el ámbito de la cultura en comparación a otras áreas de interés nacional, debido a que contempla diversas formas de financiamiento estatal y privado nacional e internacional.

- Financiamiento para la creación artística. Se considera imprescindible la constitución de mecanismos que regulen el acceso a recursos económicos, ya sea a través de fondos concursables estatales o de una vía más expedita de vinculación con privados que quieran financiar proyectos artístico-culturales.

- Inversión en difusión, circulación de las obras y formación de audiencia. Se considera necesario que el Estado invierta tanto en la difusión como circulación de obras.

Por último, los grupos focales enfatizan la participación como consumo y financiamiento, generando procesos de oferta-mercado para la formación de audiencias y difusión, así como para el financiamiento. Sin embargo, en este proceso por medio del cual se obtienen remuneraciones económicas de parte del Estado y/o privados para generar audiencia (participativa) y creación artística, puede también perderse el fondo de la cultura y el arte e incluso quitarle valor.

Desde la Unión de Artistas, enfatizan que las modificaciones a la Ley Valdés, deberían incorporar indicaciones que permitan apoyar todo tipo de obras artísticas, así como transparentar y poner a disposición la información respecto de las donaciones culturales. También se enfatiza en la eliminación de las prohibiciones u obligaciones que desalientan los aportes privados, sin derivar en un debilitamiento de las responsabilidades del Estado; en el financiamiento adecuado de las instituciones que cumplen roles importantes; establecer un programa público de difusión; fortalecer los mecanismos estatales de financiamiento a gremios, sindicatos e instituciones artísticas; y, por último, la creación de instancias de detección de talentos. 


\section{Conclusiones}

Podemos identificar que el ámbito cultural en Chile es complejo, ya que está constituido por diversos órganos públicos y privados, que asumen distintos roles y detentan distintas competencias, capacidades y recursos.

La creación del CNCA el año 2003 contempla la participación de la sociedad civil en el diseño de las políticas culturales, ya que cuenta con Consejos de la Sociedad Civil con carácter consultivo y autónomo y con el Directorio Nacional con carácter resolutivo. Esta participación es un rasgo innovador, resituando el rol del Estado, haciéndolo asumir un rol colaborativo, que reconoce la existencia de múltiples actores, con diferentes roles, competencias, capacidades y recursos.

La estructura institucional del CNCA en el gobierno de Sebastián Piñera, permite observar que el Consejo está liderado por el Ministro Presidente, evidenciando una resistencia cultural y organizativa a asumir la nueva configuración del Consejo, que contempla un carácter pluripersonal con altos grados de participación de la sociedad civil. Se observa también que en este último período hubo múltiples modificaciones estructurales al Servicio, las que dicen relación con una mayor departamentalización y especialización de las unidades de trabajo creadas, no propiciando estructuras más planas que permiten mayor comunicación entre las partes.

De esta forma, se concluye que existe una escasa participación de la sociedad civil en el Directorio, producto del debilitamiento de los consejeros y el rol pasivo que asumen frente a las intervenciones del Ministro Presidente. Este rol pasivo puede atribuirse a las reiteradas solicitudes para contar con información oportuna para la toma de decisiones, así como a la tensión que existe por el reconocimiento de las atribuciones y competencias de los consejeros, y a la falta de una estrategia comunicacional de vocería como CNCA que considerara al Directorio como parte integrante de los acuerdos adoptados. Se observa, por lo tanto, que tanto la Administración como los Consejeros presentan dificultades para adaptarse a esta nueva forma de gobernar.

Las instancias con más espacio para la reflexión y la deliberación son la Convención Nacional y los Grupos Focales. La primera está constituida en un $99 \%$ por la institucionalidad cultural, sean estos los órganos colegiados nacionales, regionales y sectoriales. La segunda incorpora una mayor diversidad como Organizaciones sin fines de lucro, academia y grupos de influencia, no obstante los participantes convocados fueron proporcionados por el CNCA.
La mayoría de las instancias de participación, con excepción de la Cuenta Pública, abogan por un proceso de definición de política pública bottom-up. Los puntos de mayor encuentro son el fomento de los procesos de circulación interregional de productos y manifestaciones artísticos culturales, la promoción de formación para la apreciación de actividades artísticas culturales, la promoción de procesos formativos para la creación destinado a niños y jóvenes desde el ámbito escolar en coordinación con el sector gubernamental correspondiente y por último el incremento de instancias de financiamiento público-privado.

Con respecto a los grupos focales, estos abogan por la descentralización, entendiendo que la participación es el mejor mecanismo para diseñar, construir, ejecutar y evaluar la política cultural. En términos de gobernanza multinivel abogan por un diálogo directo entre el CNCA y el Ministerio de Educación, ya que la labor de ambas entidades de manera coordinada y colaborativa debiera sentar las bases del cambio en los valores y apreciación respecto del patrimonio cultural nacional y, sobretodo, del cambio en las futuras generaciones.

$\mathrm{Al}$ revisar las actas del Directorio, se observa que desde el año 2006 los distintos actores se han ido diversificando e individualizando y el rol del Estado se ha resituado, dando paso a un gobierno en red. Sin embargo, en la práctica a la Administración le cuesta reconocer la presencia de la sociedad civil, sus atribuciones y facultades a nivel político y su aporte al proceso de definición de políticas culturales. Con respecto a la gobernanza multinivel, se observa que el proceso de definición de las políticas culturales sigue siendo centralizado.

Con respecto a la articulación de redes públicoprivadas, el énfasis está puesto en la figura del "gestor cultural", agente al que se le asocia el concepto de eficiencia en el ámbito cultural, teniendo la responsabilidad de generar interacciones entre los distintos actores en un marco de financiamiento mixto. Se le atribuye la capacidad de articular, coordinar y activar una estructura horizontal de relaciones

\section{Recomendaciones}

Se recomienda generar un levantamiento de información a nivel de Estado sobre todas las fuentes públicas y privadas de financiamiento disponibles, determinando así quiénes son los beneficiarios de tales aportes y presentarlo de manera consolidada a la comunidad con dos fines: por un lado, transparentar las fuentes y usos de los recursos; y por otro, poner al alcance de la ciudadanía las distintas op- 
ciones existentes. Estas medidas incrementarían el nivel de credibilidad que existe en este ámbito y la legitimidad en la asignación de recursos culturales.

Es relevante potenciar las redes como mecanismo de articulación entre los distintos actores, que puede establecerse en distintas dimensiones: inter-gubernamentales, o de gobernanza multinivel; inter-organizativas horizontales o de intersectorialidad y público-privada y participativas. Deben identificarse los roles y capacidades que los distintos actores poseen, de tal manera de poder diseñar proyectos en forma conjunta.

Es necesario resguardar la selección de los actores en los procesos, atendiendo no solo a su representatividad sino a su significatividad. Debe abrirse la institucionalidad participativa organizada, generando espacios para los grupos sociales o personas no organizados; propiciando una mayor proximidad y visibilidad de la comunidad y fortaleciendo el ámbito local municipal, mediante el modelamiento de nuevas competencias que permitan trabajar a este nivel sectores claves de desarrollo cultural.

Se recomienda también relevar el rol de CNCA, en cuanto hacer referente de opinión y credibilidad en el ámbito cultural y las artes, potenciando su interrelación con los demás agentes que se vinculan al sector, de manera tal que la cultura cruce transversalmente el Sector Público.

\section{Referencias}

Arntz, E. y Soto (2009). Participación Ciudadana para la Modernización del Estado: Diagnósticos y propuestas. En Un Mejor Estado para Chile: Propuestas de Modernización y Reformas, pp. 731-765. Andros Impresores, Santiago.

Blanco, I. y Gomá, R. (2006). Del gobierno a la gobernanza: oportunidades y retos de un nuevo paradigma. Politika, (2):11-27.

Brugué, J. y Gallego, R. (2001). Â¿Una administración pública democrática? En Ciudadanos y decisiones públicas, pp. 43-58. Ariel, Barcelona.

Consejo Nacional de la Cultura y las Artes (2005). Definiciones de Política Cultural 20052010. Technical report, Santiago.
Consejo Nacional de la Cultura y las Artes (2010). Política cultural 2011-2016: La voz de los artistas. Technical report, Santiago.

De la Maza, G. (2010). Construcción democrática, participación ciudadana y políticas públicas en Chile. Tesis doctoral, Universidad de Leiden.

Gentes, I. (2006). Modernización del Estado y Gestión Pública participativa en Chile: Alcances, Obstáculos y Perspectivas Revista América Latina. Revista América Latina Hoy, (42):61-91.

Jouve, B. (2004). La Democracia Local: Entre el Espejismo Neotocquevillista y la Globalización. Revista de Ciencia Política, XXIV(2):116-132.

Kooiman, J. (1993). Modern governance: new government-society interactions. SAGE, California.

Krippendorff, K. y Wolfson, L. (1990). Metodología de análisis de contenido: teoría y práctica. Paidós, Barcelona.

Martínez Villarroel, M. E. (2004). Ejercicios del poder político en los gobiernos loscales de Argentina. Trabajo presentado en el Cuarto Congreso Uruguayo de Ciencia Política, "La Ciencia Política desde el Sur", Asociación Uruguaya de Ciencia Política, 14-16 de noviembre de 2012.

Mayntz, R. (2001). El Estado y la Sociedad civil en la gobernanza moderna. Reforma y Democracia, (21):1-8.

Muñoz del Campo, N. (2011). La Reforma Cultural en Chile: Un ejemplo de polarización en la gestión de una Política Pública. Revista Estado, Gobierno, Gestión Pública, (18):61-91.

Natera, A. (2004). La noción de gobernanza como gestión pública participativa y reticular. Política y Gestión, (2):3-30.

Peters, G. (1995). Modelos alternativos del proceso de la política pública: de abajo hacia arriba o de arriba hacia abajo. Gestión y Política Pública, (2):257-276.

Rietbergen-McCracken, J. y Narayan-Parker, D., editores (1998). Participation and social assessment: tools and techniques, volumen 1. World Bank Publications. 